\title{
Regularity of Inhomogeneous Quasi-Linear Equations on the Heisenberg Group
}

\author{
Shirsho Mukherjee ${ }^{1}$ and Yannick Sire ${ }^{2, *}$ \\ ${ }^{1}$ Department of Mathematics, University of Bologna, Piazza di Porta San Donato 5, \\ 40126-Bologna (BO), Italy \\ 2 Department of Mathematics, Johns Hopkins University, 3400 N. Charles Street, \\ Baltimore MD 21218, USA
}

Received 9 September 2020; Accepted (in revised version) 3 October 2020

\begin{abstract}
We establish Hölder continuity of the horizontal gradient of weak solutions to quasi-linear $p$-Laplacian type non-homogeneous equations in the Heisenberg Group.
\end{abstract}

Key Words: Non-homogeneous.

AMS Subject Classifications: 35J62, 35J92, 35H20, 35R03

\section{Introduction}

The $C^{1, \alpha}$ regularity of the $p$-Laplacian has been established earlier in, for instance $[6,15,19]$ in the Euclidean setting. Its sub-elliptic analogue for homgeneous sub-elliptic equations of $p$-Laplacian type on the Heisenberg group, was unavailable until $[17,21]$, in the last years. It is therefore natural to consider the case of regularity for the corresponding inhomogeneous equation and this is the purpose of the present contribution.

In this paper, we consider the equation

$$
-\operatorname{div}_{H} a(x, \mathfrak{X} u)=\mu \quad \text { in } \Omega \subseteq \mathbb{H}^{n},
$$

where $\Omega$ is a domain and $\mu$ is a Radon measure with $|\mu|(\Omega)<\infty$ and $\mu\left(\mathbb{H}^{n} \backslash \Omega\right)=$ 0 ; hence Eq. (1.1) can be considered as defined in all of $\mathbb{H}^{n}$. Here we denote $\mathfrak{X} u=$ $\left(X_{1} u, \cdots, X_{2 n} u\right)$ as the horizontal gradient of $u: \Omega \rightarrow \mathbb{R}$, see Section 2 .

We shall take up the following structural assumptions throughout the paper: the continuous function $a: \Omega \times \mathbb{R}^{2 n} \rightarrow \mathbb{R}^{2 n}$ is assumed to be $C^{1}$ in the gradient variable and

*Corresponding author. Email addresses: shirsho.mukherjee2@unibo.it (S. Mukherjee), ysire1@jhu.edu (Y. Sire) 
satisfies the following structure condition for every $x, y \in \Omega$ and $z, \xi \in \mathbb{R}^{2 n}$,

$$
\begin{aligned}
& \left(|z|^{2}+s^{2}\right)^{\frac{p-2}{2}}|\xi|^{2} \leq\left\langle D_{z} a(x, z) \xi, \xi\right\rangle \leq L\left(|z|^{2}+s^{2}\right)^{\frac{p-2}{2}}|\xi|^{2}, \\
& |a(x, z)-a(y, z)| \leq L^{\prime}|z|\left(|z|^{2}+s^{2}\right)^{\frac{p-2}{2}}|x-y|^{\alpha},
\end{aligned}
$$

where $L, L^{\prime} \geq 1, s \geq 0, \alpha \in(0,1]$ and $D_{z} a(x, z)$ is a symmetric matrix for every $x \in \Omega$. The sub-elliptic $p$-Laplacian equation with measure data, given by

$$
-\operatorname{div}_{H}\left(|\mathfrak{X} u|^{p-2} \mathfrak{X} u\right)=\mu,
$$

is a prototype of Eq. (1.1) with the condition (1.2) for the case $s=0$. The weak solutions of (1.1) are defined in horizontal Sobolev space $H W^{1, p}(\Omega)$; the Lipschitz and Hölder classes, denoted by same classical notations, are defined with respect to the CC-metric $(x, y) \mapsto d(x, y)$, see Section 2 for details. We shall denote $Q=2 n+2$ as the homogeneous dimension. Now we state our main result.

Theorem 1.1. Let $u \in H W^{1, p}(\Omega)$ be a weak solution of $E q$. (1.1) with $p \geq 2$ and a $C^{1}$-function $a: \Omega \times \mathbb{R}^{2 n} \rightarrow \mathbb{R}^{2 n}$ satisfying the structure condition (1.2). If we have $\mu=f \in L_{\mathrm{loc}}^{q}(\Omega)$ for some $q>Q$, then $\mathfrak{X} u$ is locally Hölder continuous and there exists $c=c(n, p, L)>0$ and $\bar{R}=\bar{R}\left(n, p, L, L^{\prime}, \alpha, q, \operatorname{dist}\left(x_{0}, \partial \Omega\right)\right)>0$ such that for any $x_{0} \in \Omega, 0<R \leq \bar{R}$ and $x, y \in B_{R}\left(x_{0}\right) \subset \Omega$, the estimate

$$
|\mathfrak{X} u(x)-\mathfrak{X} u(y)| \leq c d(x, y)^{\gamma}\left(f_{B_{R}\left(x_{0}\right)}(|\mathfrak{X} u|+s) d x+\|f\|_{L^{q}\left(B_{R}\left(x_{0}\right)\right)}^{1 /(p-1)}\right),
$$

holds for some $\gamma=\gamma(n, p, L, \alpha, q) \in(0,1)$. In particular, if $a(x, z)$ is independent of $x$, then (1.4) holds for $\bar{R}=\bar{R}\left(n, p, L\right.$, $\left.\operatorname{dist}\left(x_{0}, \partial \Omega\right)\right)>0$ and $\gamma(n, p, L, q) \in(0,1)$.

The proof of Theorem 1.1 in this paper, relies on novel techniques introduced by Duzaar-Mingione [7] based on sharp comparison estimates of homogeneous equations with frozen coefficients, in other words, harmonic replacements. However, in the present sub-elliptic setting, one encounters extra terms coming from commutators of the horizontal vector fields which lead to estimates that are not always as strong as those in the Euclidean setting. An instance appears in Proposition 3.1 for the integral decay estimate, where the extra term in (3.1) appears unavoidably and can not be removed unlike similar integral estimates obtained previously in the Euclidean setting in $[7,16]$, see Remark 3.1. Hence, one gets a weaker integral decay estimate of the oscillation of the gradient of solutions of the in-homogeneous solution. Nevertheless, a perturbation lemma (Lemma 4.2), similar to the standard lemma of Campanato [3,9], leads to the $C^{1, \alpha}$-regularity of weak solutions of Eq. (1.1) by exploiting the high integrability of the data.

We develop necessary notations, definitions and provide previous results on subelliptic equations in Section 2. Then we prove the intermediate estimates in Section 3 and finally, we prove Theorem 1.1 in Section 4. 


\section{Preliminaries and previous results}

\subsection{The Heisenberg group}

Here we provide the definition and properties of Heisenberg group that would be useful in this paper. For more details, we refer to [2,5], etc. The Heisenberg Group, denoted by $\mathbb{H}^{n}$ for $n \geq 1$, is identified to the Euclidean space $\mathbb{R}^{2 n+1}$ with the group operation

$$
x \circ y:=\left(x_{1}+y_{1}, \cdots, x_{2 n}+y_{2 n}, t+s+\frac{1}{2} \sum_{i=1}^{n}\left(x_{i} y_{n+i}-x_{n+i} y_{i}\right)\right)
$$

for every $x=\left(x_{1}, \cdots, x_{2 n}, t\right), y=\left(y_{1}, \cdots, y_{2 n}, s\right) \in \mathbb{H}^{n}$. Thus, $\mathbb{H}^{n}$ with $\circ$ of $(2.1)$ forms a non-Abelian Lie group, whose left invariant vector fields corresponding to the canonical basis of the Lie algebra, are

$$
X_{i}=\partial_{x_{i}}-\frac{x_{n+i}}{2} \partial_{t}, \quad X_{n+i}=\partial_{x_{n+i}}+\frac{x_{i}}{2} \partial_{t}
$$

for every $1 \leq i \leq n$ and the only non zero commutator $T=\partial_{t}$. We have

$$
\left[X_{i}, X_{n+i}\right]=T \quad \text { and } \quad\left[X_{i}, X_{j}\right]=0, \quad \forall j \neq n+i,
$$

and we call $X_{1}, \cdots, X_{2 n}$ as horizontal vector fields and $T$ as the vertical vector field.

Given any scalar function $f: \mathbb{H}^{n} \rightarrow \mathbb{R}$, we denote $\mathfrak{X} f=\left(X_{1} f, \cdots, X_{2 n} f\right)$ the horizontal gradient and $\mathfrak{X X f} f=\left(X_{i}\left(X_{j} f\right)\right)_{i, j}$ as the horizontal Hessian. Also, the sub-Laplacian operator is denoted by $\Delta_{H} f=\sum_{j=1}^{2 n} X_{j} X_{j} f$. For a vector valued function $F=\left(f_{1}, \cdots, f_{2 n}\right)$ : $\mathbb{H}^{n} \rightarrow \mathbb{R}^{2 n}$, the horizontal divergence is defined as

$$
\operatorname{div}_{H}(F)=\sum_{i=1}^{2 n} X_{i} f_{i}
$$

The Euclidean gradient of a scalar function $g: \mathbb{R}^{k} \rightarrow \mathbb{R}$, shall be denoted by $\nabla g=$ $\left(D_{1} g, \cdots, D_{k} g\right)$ and the Hessian matrix by $D^{2} g$.

The Carnot-Carathèodory metric (CC-metric) is defined as the length of the shortest horizontal curves connecting two points, see [5], and is denoted by $d$. This is equivalent to the homogeneous metric, denoted as $d_{\mathbb{H}^{n}}(x, y)=\left\|y^{-1} \circ x\right\|_{\mathbb{H}^{n}}$, where the homogeneous norm for $x=\left(x_{1}, \cdots, x_{2 n}, t\right) \in \mathbb{H}^{n}$ is

$$
\|x\|_{\mathbb{H}^{n}}:=\left(\sum_{i=1}^{2 n} x_{i}^{2}+|t|\right)^{\frac{1}{2}} .
$$

Throughout this article we use the CC-metric balls $B_{r}(x)=\left\{y \in \mathbb{H}^{n}: d(x, y)<r\right\}$ for $r>0$ and $x \in \mathbb{H}^{n}$. However, by virtue of the equivalence of the metrics, all assertions for CC-balls can be restated to any homogeneous metric balls. 
The Haar measure of $\mathbb{H}^{n}$ is just the Lebesgue measure of $\mathbb{R}^{2 n+1}$. For a measurable set $E \subset \mathbb{H}^{n}$, we denote the Lebesgue measure as $|E|$. For an integrable function $f$, we denote

$$
(f)_{E}=f_{E} f d x=\frac{1}{|E|} \int_{E} f d x .
$$

The Hausdorff dimension with respect to the metric $d$ is also the homogeneous dimension of the group $\mathbb{H}^{n}$, which shall be denoted as $Q=2 n+2$, throughout this paper. Thus, for any CC-metric ball $B_{r}$, we have that $\left|B_{r}\right|=c(n) r^{Q}$.

For $1 \leq p<\infty$, the Horizontal Sobolev space $H^{1, p}(\Omega)$ consists of functions $u \in$ $L^{p}(\Omega)$ such that the distributional horizontal gradient $\mathfrak{X} u$ is in $L^{p}\left(\Omega, \mathbb{R}^{2 n}\right) . H W^{1, p}(\Omega)$ is a Banach space with respect to the norm

$$
\|u\|_{H W^{1, p}(\Omega)}=\|u\|_{L^{p}(\Omega)}+\|\mathfrak{X} u\|_{L^{p}\left(\Omega, \mathbb{R}^{2 n}\right)} .
$$

We define $H_{W_{\text {loc }}^{1, p}}(\Omega)$ as its local variant and $H_{0}^{1, p}(\Omega)$ as the closure of $C_{0}^{\infty}(\Omega)$ in $H W^{1, p}(\Omega)$ with respect to the norm in (2.4). The Sobolev Embedding theorem has the following version in the setting of Heisenberg group, see $[4,5,12]$ etc.

Theorem 2.1 (Sobolev Inequality). Given $B_{r} \subset \mathbb{H}^{n}$ and $1<q<Q$, there exists $c=c(n, q)>$ 0 such that, for every $u \in H_{0}^{1, q}\left(B_{r}\right)$ we have

$$
\left(\int_{B_{r}}|u|^{\frac{Q q}{Q-q}} d x\right)^{\frac{Q-q}{Q q}} \leq c\left(\int_{B_{r}}|\mathfrak{X} u|^{q} d x\right)^{\frac{1}{q}} .
$$

Hölder spaces with respect to homogeneous metrics have been defined in FollandStein [8] and therefore, are sometimes known as Folland-Stein classes and denoted by $\Gamma^{\alpha}$ or $\Gamma^{0, \alpha}$ in some literature. However, as in $[17,21]$, here we continue to maintain the classical notation and define

$$
C^{0, \alpha}(\Omega)=\left\{u \in L^{\infty}(\Omega):|u(x)-u(y)| \leq c d(x, y)^{\alpha}, \forall x, y \in \Omega\right\}
$$

for $0<\alpha \leq 1$, which are Banach spaces with the norm

$$
\|u\|_{C^{0, \alpha}(\Omega)}=\|u\|_{L^{\infty}(\Omega)}+\sup _{x, y \in \Omega} \frac{|u(x)-u(y)|}{d(x, y)^{\alpha}} .
$$

These have standard extensions to classes $C^{k, \alpha}(\Omega)$ for $k \in \mathbb{N}$, comprising functions having horizontal derivatives up to order $k$ in $C^{0, \alpha}(\Omega)$; their local counterparts are denoted as $C_{\text {loc }}^{k, \alpha}(\Omega)$. The Morrey embedding theorem is the following.

Theorem 2.2 (Morrey Inequality). Given any $B_{r} \subset \mathbb{H}^{n}$ and $q>Q$, there exists $c=c(n, q)>$ 0 such that, for every $u \in H W_{0}^{1, q}\left(B_{r}\right) \cap C\left(\bar{B}_{r}\right)$ we have

$$
|u(x)-u(y)| \leq c d(x, y)^{1-Q / q}\left(\int_{B_{r}}|\mathfrak{X} u|^{q} d x\right)^{\frac{1}{q}}, \quad \forall x, y \in B_{r} .
$$




\subsection{Sub-elliptic equations}

Here, we enlist some of the properties and results previously known for sub-elliptic equations of the form (1.1).

First, we recall that the structure condition (1.2) implies the monotonicity and ellipticity inequalities, as follows:

$$
\begin{aligned}
& \left\langle a\left(x, z_{1}\right)-a\left(x, z_{2}\right), z_{1}-z_{2}\right\rangle \geq c\left(\left|z_{1}\right|^{2}+\left|z_{2}\right|^{2}+s^{2}\right)^{\frac{p-2}{2}}\left|z_{1}-z_{2}\right|^{2}, \\
& \langle a(x, z), z\rangle \geq c\left(|z|^{2}+s^{2}\right)^{\frac{p-2}{2}}|z|^{2}
\end{aligned}
$$

for some $c=c(n, p, L)>0$. This ensures existence and local uniqueness of weak solution $u \in H W^{1, p}(\Omega)$ of Eq. (1.1) from the classical theory of monotone operators, see [14]. We denote $u$ as the precise representative, hereafter.

The regularity and apriori estimates of the homogeneous equation corresponding to (1.1) with freezing of the coefficients, is necessary. Therefore, for any $x_{0} \in \Omega$, we consider the equation

$$
\operatorname{div}_{H} a\left(x_{0}, \mathfrak{X} u\right)=0 \text { in } \Omega .
$$

The $C^{1, \alpha}$ regulaity of $p$-Laplacian type equations has been dealt with in $[17,21]$, where the equation $\operatorname{div}_{H}(D f(\mathfrak{X} u))=0$ has been considered. Given $D_{z} a\left(x_{0}, z\right)$ being symmetric, all the arguments there also follow in the same way for (2.10) with the growth conditions (1.2) which is the same as that in [17] and slightly weaker than that in [21] (in fact, (2.10) has been considered in [18] in a more general setting). The following regularity theorem is due to [21, Theorem 1.1] and [17, Theorem 1.3].

Theorem 2.3. If $u \in H W^{1, p}(\Omega)$ is a weak solution of $E q$. (2.10) with $a\left(x_{0}, z\right)$ satisfying the condition (1.2) and $D_{z} a\left(x_{0}, z\right)$ is a symmetric matrix, then $\mathfrak{X} u$ is locally Hölder continuous. Moreover, there exist constants $c=c(n, p, L)>0$ and $\beta=\beta(n, p, L) \in(0,1)$ such that the following holds,

$$
\begin{aligned}
& \text { (i) } \sup _{B_{R / 2}}|\mathfrak{X} u|^{p} \leq c f_{B_{R}}\left(|\mathfrak{X} u|^{2}+s^{2}\right)^{\frac{p}{2}} d x, \\
& \text { (ii) } f_{B_{\varrho}}\left|\mathfrak{X} u-(\mathfrak{X} u)_{B_{\varrho}}\right|^{p} d x \leq c(\varrho / R)^{\beta} f_{B_{R}}\left(|\mathfrak{X} u|^{2}+s^{2}\right)^{\frac{p}{2}} d x,
\end{aligned}
$$

for every concentric $B_{Q} \subseteq B_{R} \subset \Omega$ and $1<p<\infty$.

In fact, similarly as the Euclidean case, the following local estimate can be shown by using Sobolev's inequality and Moser's iteration on the Caccioppoli type inequalities of [21], for any $0<\sigma<1$ and $q>0$,

$$
\sup _{B_{\sigma R}}|\mathfrak{X} u| \leq c(1-\sigma)^{-\frac{Q}{q}}\left(f_{B_{R}}\left(|\mathfrak{X} u|^{2}+s^{2}\right)^{\frac{q}{2}} d x\right)^{\frac{1}{q}}
$$


for some $c=c(n, p, L, q)>0$, see [21, p. 12]. Thus, taking $q=1$, we can have

$$
\sup _{B_{R / 2}}|\mathfrak{X} u| \leq c f_{B_{R}}(|\mathfrak{X} u|+s) d x .
$$

From (2.13) it ie easy to see that for all $0<r \leq R / 2$, we have

$$
\int_{B_{r}}|\mathfrak{X} u| d x \leq c\left(\frac{r}{R}\right)^{Q} \int_{B_{R}}(|\mathfrak{X} u|+s) d x,
$$

where $u \in C^{1, \beta}(\Omega)$ is a solution of Eq. (2.10) in the above inequalities.

We recall the notion of De Giorgi's class of functions in this setting, which would be required for Proposition 3.1, in Section 3. Given a metric ball $B_{\rho_{0}} \subset \mathbb{H}^{n}$, the De Giorgi's class $D G^{+}\left(B_{\rho_{0}}\right)$ consists of functions $v \in H W^{1,2}\left(B_{\rho_{0}}\right) \cap L^{\infty}\left(B_{\rho_{0}}\right)$, which satisfy the inequality

$$
\int_{B_{\rho^{\prime}}}\left|\mathfrak{X}(v-k)^{+}\right|^{2} d x \leq \frac{\gamma}{\left(\rho-\rho^{\prime}\right)^{2}} \int_{B_{\rho}}\left|(v-k)^{+}\right|^{2} d x+\chi^{2}\left|A_{k, \rho}^{+}\right|^{1-\frac{2}{Q}+\epsilon}
$$

for some $\gamma, \chi, \epsilon>0$, where $A_{k, \rho}^{+}=\left\{x \in B_{\rho}:(v-k)^{+}=\max (v-k, 0)>0\right\}$ for any arbitrary $k \in \mathbb{R}$, the balls $B_{\rho^{\prime}}, B_{\rho}$ and $B_{\rho_{0}}$ are concentric with $0<\rho^{\prime}<\rho \leq \rho_{0}$. The class $D G^{-}\left(B_{\rho_{0}}\right)$ is similarly defined and $D G\left(B_{\rho_{0}}\right)=D G^{+}\left(B_{\rho_{0}}\right) \cap D G^{-}\left(B_{\rho_{0}}\right)$. All properties of classical De Giorgi class functions, also hold for these classes.

We end this section by introducing the sub-elliptic Wolff potential given by

$$
\boldsymbol{W}_{\beta, p}^{\mu}\left(x_{0}, R\right):=\int_{0}^{R}\left(\frac{|\mu|\left(B_{\varrho}\left(x_{0}\right)\right)}{\varrho^{Q-\beta p}}\right)^{\frac{1}{p-1}} \frac{d \varrho}{\varrho}, \quad \forall \beta \in(0, Q / p],
$$

and recalling following lemma of the density of Wolff potential, see [7] for proof.

Lemma 2.1. Given any $H>1, x_{0} \in \Omega$ and $r>0$, if $r_{i}=r / H^{i}$ for every $i \in\{0,1,2, \cdots\}$, then we have

$$
\sum_{i=0}^{\infty}\left(\frac{|\mu|\left(B_{r_{i}}\left(x_{0}\right)\right)}{r_{i}^{Q-1}}\right)^{\frac{1}{p-1}} \leq\left(\frac{2^{\frac{Q-1}{p-1}}}{\log (2)}+\frac{H^{\frac{Q-1}{p-1}}}{\log (H)}\right) \boldsymbol{W}_{\frac{1}{p}, p}^{\mu}\left(x_{0}, 2 r\right) .
$$

\section{Estimates of the horizontal gradient}

In this section, we show several comparison estimates along the lines of $[7,13]$ ultimately leading to a pointwise estimate of the horizontal gradient. Here onwards we fix $x_{0} \in \Omega$ and denote $B_{\varrho}=B_{\varrho}\left(x_{0}\right)$ for every $\varrho>0$. Also, we denote all constants as $c$, the values of which may vary from line to line but they are positive and dependent only on $n, p, L$, unless explicitly specified otherwise.

In the following, first we show the integral oscillation decay estimate of solutions of Eq. (2.10), analogous to that of the Euclidean setting in $[7,16]$ etc. 
Proposition 3.1. Let $B_{r_{0}} \subset \Omega$ and $u \in C^{1, \beta}(\Omega)$ be a solution of $E q$. (2.10), with $\beta=$ $\beta(n, p, L) \in(0,1)$. Then there exists $c=c(n, p, L)>0$, such that for all $0<\varrho<r<r_{0}$, we have

$$
f_{B_{\varrho}}\left|\mathfrak{X} u-(\mathfrak{X} u)_{B_{\varrho}}\right| d x \leq c\left(\frac{\varrho}{r}\right)^{\beta}\left[f_{B_{r}}\left|\mathfrak{X} u-(\mathfrak{X} u)_{B_{r}}\right| d x+\chi r^{\beta}\right]
$$

with $\chi=\left(s+M\left(r_{0}\right)\right) / r_{0}^{\beta}$, where

$$
M\left(r_{0}\right)=\max _{1 \leq i \leq 2 n} \sup _{B_{r_{0}}}\left|X_{i} u\right| .
$$

Proof. Given $B_{r_{0}} \subset \Omega$, let us denote

$$
M(\rho)=\max _{1 \leq i \leq 2 n} \sup _{B_{\rho}}\left|X_{i} u\right|
$$

and

$$
\omega(\rho)=\max _{1 \leq i \leq 2 n} \operatorname{osc}_{B_{\rho}} X_{i} u \text { and } I(\rho)=f_{B_{\rho}}\left|\mathfrak{X} u-(\mathfrak{X} u)_{B_{\rho}}\right| d x
$$

for every $0<\rho<r_{0}$. Hence, note that $\omega(\rho) \leq 2 M(\rho)$. Now, we recall the oscillation lemma proved in [17, Theorem 4.1], that there exists $m=m(n, p, L) \geq 0$ such that for every $0<r \leq r_{0} / 16$, we have

$$
\omega(r) \leq\left(1-2^{-m}\right) \omega(8 r)+2^{m}\left(s+M\left(r_{0}\right)\right)\left(\frac{r}{r_{0}}\right)^{\beta},
$$

for some $\beta=\beta(n, p, L) \in(0,1 / p)$. A standard iteration on (3.3), see for instance [11, Lemma 7.3], implies that for every $0<\varrho<r \leq r_{0}$, we have

$$
\omega(\varrho) \leq c\left[\left(\frac{\varrho}{r}\right)^{\beta} \omega(r)+\chi \varrho^{\beta}\right]=c\left(\frac{\varrho}{r}\right)^{\beta}\left[\omega(r)+\chi r^{\beta}\right],
$$

where $\chi=\left(s+M\left(r_{0}\right)\right) / r_{0}^{\beta}$ and $c=c(n, p, L)>0$. If $\varrho \leq \delta r$ for some $\delta \in(0,1)$, it is easy to see from (3.4), that for some $c=c(n, p, L)>0$, we have

$$
I(\varrho) \leq c \omega(\varrho) \leq c \delta^{-\beta}\left(\frac{\varrho}{r}\right)^{\beta}\left[\omega(\delta r)+\chi r^{\beta}\right] .
$$

Now we claim that, there exists $\delta=\delta(n, p, L) \in(0,1)$ such that, the inequality

$$
\omega(\delta r) \leq c\left[I(r)+\chi r^{\beta}\right]
$$

holds for some $c=c(n, p, L)>0$. Then (3.5) and (3.6) together, yields (3.1); hence proving the claim (3.6) is enough to complete the proof.

To this end, let us denote $r^{\prime}=\delta r$, where $\delta \in(0,1)$ is to be chosen later. Notice that, to prove the claim (3.6), we can make the priori assumption:

$$
\omega(r) \geq\left(s+M\left(r_{0}\right)\right)\left(r / r_{0}\right)^{\sigma},
$$


with $\sigma=1 / p$ for $p \geq 2$, since, otherwise (3.6) holds trivially with $\beta=\sigma$. Now, we consider the following complementary cases. This is very standard for elliptic estimates, see $[6,7,16,19]$ for corresponding Euclidean cases.

Case 1: For at least one index $l \in\{1, \cdots, 2 n\}$, we have either

$$
\left|B_{4 r^{\prime}} \cap\left\{X_{l} u<\frac{M\left(4 r^{\prime}\right)}{4}\right\}\right| \leq \theta\left|B_{4 r^{\prime}}\right| \quad \text { or } \quad\left|B_{4 r^{\prime}} \cap\left\{X_{l} u>-\frac{M\left(4 r^{\prime}\right)}{4}\right\}\right| \leq \theta\left|B_{4 r^{\prime}}\right| .
$$

It has been shown in [17, Theorem 4.1] that under assumption (3.7), if Case 1 holds with choice of a small enough $\theta=\theta(n, p, L)>0$, then $X_{i} u \in D G\left(B_{2 r^{\prime}}\right)$ for every $i \in\{1, \cdots, 2 n\}$. Then, the standard local boundedness estimates of De Giorgi class functions [11, Theorems 7.2 and 7.3] follow; the fact that $X_{i} u$ belongs to $D G^{+}\left(B_{2 r^{\prime}}\right)$ and $D G^{-}\left(B_{2 r^{\prime}}\right)$, yields the following respective estimates for any $\vartheta<M\left(r^{\prime}\right)$ :

$$
\begin{aligned}
& \sup _{B_{r^{\prime}}}\left(X_{i} u-\vartheta\right) \leq c\left[f_{B_{2 r^{\prime}}}\left(X_{i} u-\vartheta\right)^{+} d x+\chi r^{\prime \beta}\right], \\
& \sup _{B_{r^{\prime}}}\left(\vartheta-X_{i} u\right) \leq c\left[f_{B_{2 r^{\prime}}}\left(\vartheta-X_{i} u\right)^{+} d x+\chi r^{\prime \beta}\right],
\end{aligned}
$$

for every $i \in\{1, \cdots, 2 n\}$. Adding (3.8a) and (3.8b) with $\vartheta=\left(X_{i} u\right)_{B_{r^{\prime}}}$, we get

$$
\operatorname{osc}_{B_{r^{\prime}}} X_{i} u \leq c\left[f_{B_{2 r^{\prime}}}\left|X_{i} u-\left(X_{i} u\right)_{B_{r^{\prime}}}\right| d x+\chi r^{\prime \beta}\right] \leq c\left[I(r)+\chi r^{\beta}\right]
$$

for some $c=c(n, p, L)>0$ and $\delta<1 / 2$, which further implies (3.6) for this case.

Case 2: With $\theta=\theta(n, p, L)>0$ as in Case 1, for every $i \in\{1, \cdots, 2 n\}$, we have

$$
\left|B_{4 r^{\prime}} \cap\left\{X_{i} u<\frac{M\left(4 r^{\prime}\right)}{4}\right\}\right|>\theta\left|B_{4 r^{\prime}}\right| \text { and }\left|B_{4 r^{\prime}} \cap\left\{X_{i} u>-\frac{M\left(4 r^{\prime}\right)}{4}\right\}\right|>\theta\left|B_{4 r^{\prime}}\right| .
$$

First, we notice that the above assertions respectively imply $\inf _{B_{4 r^{\prime}}} X_{i} u \leq M\left(4 r^{\prime}\right) / 4$ and $\sup _{B_{4 r^{\prime}}} X_{i} u \geq-M\left(4 r^{\prime}\right) / 4$ for every $i \in\{1, \cdots, 2 n\}$. These further imply that

$$
\omega\left(4 r^{\prime}\right) \geq M\left(4 r^{\prime}\right)-M\left(4 r^{\prime}\right) / 4=3 M\left(4 r^{\prime}\right) / 4 \text {. }
$$

Now, let us denote

$$
L=\max _{1 \leq i \leq 2 n}\left|\left(X_{i} u\right)_{B_{r}}\right|=\left|\left(X_{k} u\right)_{B_{r}}\right|
$$

for some $k \in\{1, \cdots, 2 n\}$. Then note that, if $L>2 \omega\left(4 r^{\prime}\right)$ then using (3.9), we have

$$
\left|\left(X_{k} u\right)_{B_{r}}\right|-\left|X_{k} u\right| \geq 2 \omega\left(4 r^{\prime}\right)-M\left(4 r^{\prime}\right) \geq M\left(4 r^{\prime}\right) / 2 \text { in } B_{4 r^{\prime}},
$$

which, together with the choice of $\delta<1 / 4$, further implies

$$
I(r) \geq c(n) f_{B_{4 r^{\prime}}}\left|X_{k} u-\left(X_{k} u\right)_{B_{r}}\right| d x \geq \frac{c(n)}{2} M\left(4 r^{\prime}\right) \geq \frac{c(n)}{4} \omega\left(4 r^{\prime}\right) .
$$


If $L \leq 2 \omega\left(4 r^{\prime}\right)=2 \omega(4 \delta r)$ then, we choose $\delta<1 / 8$, so that using $\omega(r / 2) \leq 2 M(r / 2)$ and (2.13) i.e.,

$$
M(r / 2) \leq c f_{B_{r}}|\mathfrak{X} u| d x,
$$

respectively on (3.4), we obtain

$$
\begin{aligned}
\omega(4 \delta r) & \leq c(8 \delta)^{\beta}\left[\omega(r / 2)+\chi r^{\beta}\right] \leq c \delta^{\beta}\left[f_{B_{r}}|\mathfrak{X} u| d x+\chi r^{\beta}\right] \\
& \leq c_{1} \delta^{\beta}\left[I(r)+L+\chi r^{\beta}\right] \leq c_{1} \delta^{\beta}\left[I(r)+2 \omega(4 \delta r)+\chi r^{\beta}\right]
\end{aligned}
$$

for some $c_{1}=c_{1}(n, p, L)>0$, where the second last inequality of the above is a consequence of triangle inequality and the definition of $I$ and $L$. Now we make a further reduction of $\delta$, such that $2 c_{1} \delta^{\beta}<1$, so that (3.11) imply

$$
\omega(4 \delta r) \leq \frac{c_{1} \delta^{\beta}}{1-2 c_{1} \delta^{\beta}}\left[I(r)+\chi r^{\beta}\right] .
$$

Thus (3.10) and (3.12) together shows that (3.6) holds for Case 2, as well. Therefore, we have shown that claim (3.6) holds for both cases and the proof is finished.

Remark 3.1. For the Euclidean case, i.e.,

$$
\operatorname{div}\left(|\nabla u|^{p-2} \nabla u\right)=0,
$$

it is well known, see $[7,16]$, that for any $0<\varrho<r$, the following estimate holds:

$$
f_{B_{\varrho}}\left|\nabla u-(\nabla u)_{B_{e}}\right| d x \leq c\left(\frac{\varrho}{r}\right)^{\beta} f_{B_{r}}\left|\nabla u-(\nabla u)_{B_{r}}\right| d x .
$$

The purpose of the Proposition 3.1 is to show that the sub-elliptic setting is very different even for the homogeneous equation and the integral oscillation estimate is not as strong as the above. We have the extra term $\chi \neq 0$ in (3.1) which one can not get rid of from estimates in $[17,21]$. Its source goes back to the extra terms containing the commutator $T u=\left[X_{i} u, X_{n+i} u\right]$ in the De Giorgi type estimates of $[17,21]$, where $T u$ is locally majorized by $\mathfrak{X} u$ from an integrability estimate in [21].

Thus, if $u \in C^{1, \beta}(\Omega)$ is a solution of Eq. (2.10), the integral decay estimate of the oscillation we end up with from (2.13) and (3.1), is

$$
f_{B_{\varrho}}\left|\mathfrak{X} u-(\mathfrak{X} u)_{B_{\varrho}}\right| d x \leq c\left(\frac{\varrho}{r}\right)^{\beta} f_{B_{r}}(|\mathfrak{X} u|+s) d x,
$$

for any $0<\varrho<r$, which is not as strong as the (3.13). Nevertheless, it is good enough for proving Theorem 1.1 via a perturbation argument. 


\subsection{Comparison estimates}

In this subsection, we prove comparison estimates essential for the proof of our theorems, by localizing Eqs. (1.1) and (2.10). They follow similarly as in the Euclidean case in [7]. Here onwards, we denote $u \in H W^{1, p}(\Omega)$ as a weak solution of (1.1) and $p \geq 2$.

Fix $R>0$ such that $B_{2 R} \subset \Omega$ and consider the Dirichlet problem

$$
\left\{\begin{array}{l}
\operatorname{div}_{H} a(x, \mathfrak{X} w)=0 \quad \text { in } B_{2 R}, \\
w-u \in H W_{0}^{1, p}\left(B_{2 R}\right) .
\end{array}\right.
$$

The following is the first comparison lemma where the density of the Wolff potential (2.16) appears in the estimates. The proof is similar to that of [7], see also [1].

Lemma 3.1. Let $u \in H W^{1, p}(\Omega)$ be a weak solution of Eq. (1.1) and $p \geq 2$. Then, the weak solution $w \in H W^{1, p}\left(B_{2 R}\right)$ of Eq. (3.15) satisfies

$$
f_{B_{2 R}}|\mathfrak{X} w-\mathfrak{X} u| d x \leq c\left(\frac{|\mu|\left(B_{2 R}\right)}{R^{Q-1}}\right)^{\frac{1}{p-1}},
$$

for some $c=c(n, p, L)>0$.

Proof. By testing Eq. (3.15) with $\varphi \in H W_{0}^{1, p}\left(B_{2 R}\right)$ and using Eq. (1.1), we have the weak formulation

$$
\int_{B_{2 R}}\langle a(x, \mathfrak{X} u)-a(x, \mathfrak{X} w), \mathfrak{X} \varphi\rangle d x=\int_{B_{2 R}} \varphi d \mu,
$$

which we estimate with appropriate choices of $\varphi$, in order to show (3.16).

First, we assume $2 \leq p \leq Q$. For any $j \in \mathbb{N}$, we denote the following truncations

$$
\begin{aligned}
& \psi_{j}=\max \left\{-\frac{j}{R \gamma}, \min \left\{\frac{u-w}{m}, \frac{j}{R^{\gamma}}\right\}\right\}, \\
& \varphi_{j}=\max \left\{-\frac{1}{R^{\gamma}}, \min \left\{\frac{u-w}{m}-\psi_{j}, \frac{1}{R^{\gamma}}\right\}\right\},
\end{aligned}
$$

where the scaling constants $m, \gamma \geq 0$ are to be chosen later. Notice that, for each $j \in \mathbb{N}$, we have

$$
\left|\varphi_{j}\right| \leq 1 / R^{\gamma} \quad \text { and } \quad \mathfrak{X} \varphi_{j}=\frac{1}{m}(\mathfrak{X} u-\mathfrak{X} w) \mathbb{1}_{E_{j}},
$$

where

$$
E_{j}=\left\{m j / R^{\gamma}<|u-w| \leq m(j+1) / R^{\gamma}\right\} .
$$

Taking $\varphi=\varphi_{j}$ in (3.17) and using (2.9a) with $p \geq 2$, it is easy to obtain

$$
\int_{B_{2 R} \cap E_{j}}|\mathfrak{X} w-\mathfrak{X} u|^{p} d x \leq \frac{c m}{R^{\gamma}}|\mu|\left(B_{2 R}\right)
$$


for some $c=c(n, p, L)>0$. Now, using Hölder's inequality and (3.18), we obtain

$$
\begin{aligned}
\int_{B_{2 R} \cap E_{j}}|\mathfrak{X} w-\mathfrak{X} u| d x & \leq\left|E_{j}\right|^{\frac{p-1}{p}}\left(\int_{B_{2 R} \cap E_{j}}|\mathfrak{X} w-\mathfrak{X} u|^{p} d x\right)^{\frac{1}{p}} \\
& \leq c\left|E_{j}\right|^{\frac{p-1}{p}}\left(m / R^{\gamma}\right)^{\frac{1}{p}}|\mu|\left(B_{2 R}\right)^{\frac{1}{p}},
\end{aligned}
$$

then, using the fact that $|u-w|^{\kappa}>\left(m j / R^{\gamma}\right)^{\kappa}$ in $E_{j}$, we obtain

$$
\int_{B_{2 R} \cap E_{j}}|\mathfrak{X} w-\mathfrak{X} u| d x \leq \frac{c\left(m / R^{\gamma}\right)^{\frac{1}{p}}|\mu|\left(B_{2 R}\right)^{\frac{1}{p}}}{\left(m j / R^{\gamma}\right)^{\frac{\kappa(p-1)}{p}}}\left(\int_{B_{2 R} \cap E_{j}}|u-w|^{\kappa} d x\right)^{\frac{p-1}{p}}
$$

with $\kappa=Q /(Q-1)$. Also from (3.18), note that for any $N \in \mathbb{N}$,

$$
\begin{aligned}
\int_{B_{2 R} \cap\left\{|u-w| \leq m N / R^{\gamma}\right\}}|\mathfrak{X} w-\mathfrak{X} u|^{p} d x & =\sum_{j=0}^{N-1} \int_{B_{2 R} \cap E_{j}}|\mathfrak{X} w-\mathfrak{X} u|^{p} d x \\
& \leq \frac{c m}{R^{\gamma}} N|\mu|\left(B_{2 R}\right) .
\end{aligned}
$$

Now, we estimate the whole integral using (3.20) and (3.19), as follows:

$$
\begin{aligned}
& \int_{B_{2 R}}|\mathfrak{X} w-\mathfrak{X} u| d x \\
= & \int_{B_{2 R} \cap\left\{|u-w| \leq m N / R^{\gamma}\right\}}|\mathfrak{X} w-\mathfrak{X} u| d x+\int_{B_{2 R} \cap\left\{|u-w|>m N / R^{\gamma}\right\}}|\mathfrak{X} w-\mathfrak{X} u| d x \\
\leq & \left|B_{2 R}\right|^{\frac{p-1}{p}}\left(\int_{B_{2 R} \cap\{|u-w| \leq m N / R \gamma\}}|\mathfrak{X} w-\mathfrak{X} u|^{p} d x\right)^{\frac{1}{p}}+\sum_{j=N}^{\infty} \int_{B_{2 R} \cap E_{j}}|\mathfrak{X} w-\mathfrak{X} u| d x \\
\leq & c\left(m / R^{\gamma}\right)^{\frac{1}{p}}|\mu|\left(B_{2 R}\right)^{\frac{1}{p}}\left(\left|B_{2 R}\right|^{\frac{p-1}{p}} N^{\frac{1}{p}}+\sum_{j=N}^{\infty}\left[\frac{1}{(m j / R \gamma)^{\kappa}} \int_{B_{2 R} \cap E_{j}}|u-w|^{\kappa} d x\right]^{\frac{p-1}{p}}\right) .
\end{aligned}
$$

Using Sobolev inequality (2.5) on the second term of the above, we obtain

$$
\begin{aligned}
& \quad \int_{B_{2 R}}|\mathfrak{X} w-\mathfrak{X} u| d x \\
& \leq c\left(m / R^{\gamma}\right)^{\frac{1}{p}}|\mu|\left(B_{2 R}\right)^{\frac{1}{p}}\left|B_{2 R}\right|^{\frac{p-1}{p}} N^{\frac{1}{p}} \\
& \quad+c\left(m / R^{\gamma}\right)^{\frac{1}{p}-\frac{\kappa(p-1)}{p}}|\mu|\left(B_{2 R}\right)^{\frac{1}{p}} \epsilon(N)^{\frac{1}{p}}\left(\int_{B_{2 R}}|\mathfrak{X} u-\mathfrak{X} w| d x\right)^{\frac{\kappa(p-1)}{p}},
\end{aligned}
$$

where

$$
\epsilon(N)=\sum_{j=N}^{\infty} 1 / j^{\kappa(p-1)}, \quad \kappa=Q /(Q-1) \quad \text { and } \quad c=c(n, p, L)>0
$$


Now, first we consider the case $p<Q$, so that we have $\kappa(p-1) / p<1$. Then, by applying Young's inequality on the second term, we obtain

$$
\begin{gathered}
\int_{B_{2 R}}|\mathfrak{X} w-\mathfrak{X} u| d x \leq c\left(\frac{m}{R \gamma}\right)^{\frac{1}{p}}|\mu|\left(B_{2 R}\right)^{\frac{1}{p}}\left|B_{2 R}\right|^{\frac{p-1}{p}} N^{\frac{1}{p}}+c\left(\frac{m}{R \gamma}\right)^{\frac{1+\kappa-\kappa p}{p+\kappa \kappa p}}|\mu|\left(B_{2 R}\right)^{\frac{1}{p+\kappa-\kappa p}} \\
+\epsilon(N)^{\frac{1}{\kappa(p-1)}}\left(\int_{B_{2 R}}|\mathfrak{X} u-\mathfrak{X} w| d x\right)
\end{gathered}
$$

for some $c=c(n, p, L)>0$. Now, we make the following choice of the scaling constants,

$$
m=|\mu|\left(B_{2 R}\right)^{\frac{1}{p-1}} \text { and } \gamma=(Q-p) /(p-1),
$$

such that the first two terms of the above are the same. Also note that, since $p \geq 2>$ $1+1 / \kappa$, we have $\kappa(p-1)>1$ and hence,

$$
\sum_{j=1}^{\infty} 1 / j^{\kappa(p-1)}=\zeta(\kappa(p-1))<\infty .
$$

If $N$ is large enough, we can have

$$
\epsilon(N)=\sum_{j=N}^{\infty} 1 / j^{\kappa(p-1)}<1 / 2^{\kappa(p-1)}
$$

and thus, the last term of the estimate can be absolved in the left hand side. With these choices of $m, \gamma, N$, we finally obtain

$$
\int_{B_{2 R}}|\mathfrak{X} w-\mathfrak{X} u| d x \leq c|\mu|\left(B_{2 R}\right)^{\frac{1}{p-1}} R^{\frac{Q p-2 Q+1}{p-1}}
$$

for some $c=c(n, p, L)>0$, which immediately implies (3.16).

For the case of $p=Q$, the estimate (3.21) also follows similary with a possibly larger $N$ and the same choices of scaling constants, i.e.,

$$
m=|\mu|\left(B_{2 R}\right)^{1 /(Q-1)} \quad \text { and } \quad \gamma=0,
$$

except here we absolve the last term to the right hand side directly, without using Young's inequality.

Now we assume the $p \geq Q$. Here we simply choose $\varphi=u-w$ in (3.17) and use (2.9a) together with Morrey's inequality (2.8) to obtain

$$
\begin{aligned}
\int_{B_{2 R}}|\mathfrak{X} w-\mathfrak{X} u|^{p} d x & \leq c \int_{B_{2 R}}|u-w| d \mu \leq c|\mu|\left(B_{2 R}\right) \sup _{B_{2 R}}|u-w| \\
& \leq c|\mu|\left(B_{2 R}\right) R^{1-\frac{Q}{p}}\left(\int_{B_{2 R}}|\mathfrak{X} w-\mathfrak{X} u|^{p} d x\right)^{\frac{1}{p}},
\end{aligned}
$$


which, upon using Young's inequality, yields

$$
\int_{B_{2 R}}|\mathfrak{X} w-\mathfrak{X} u|^{p} d x \leq c|\mu|\left(B_{2 R}\right)^{\frac{p}{p-1}} R^{\frac{p-Q}{p-1}} .
$$

Then, using Hölder's inequality and (3.22), we obtain

$$
\begin{aligned}
\int_{B_{2 R}}|\mathfrak{X} w-\mathfrak{X} u| d x & \leq\left|B_{2 R}\right|^{\frac{p-1}{p}}\left(\int_{B_{2 R}}|\mathfrak{X} w-\mathfrak{X} u|^{p} d x\right)^{\frac{1}{p}} \\
& \leq c|\mu|\left(B_{2 R}\right)^{\frac{1}{p-1}} R^{\frac{Q p-2 Q+1}{p-1}}
\end{aligned}
$$

which, just as before, implies (3.16). Thus, the proof is finished.

Remark 3.2. It is evident that by using Sobolev or Morrey inequality (2.5), (2.8) on (3.16), we can obtain the estimate

$$
f_{B_{2 R}}|w-u| d x \leq c\left(\frac{|\mu|\left(B_{2 R}\right)}{R^{Q-p}}\right)^{\frac{1}{p-1}}
$$

where $u$ and $w$ are the functions stated in Lemma 3.1.

For the next comparison estimate, we require the Dirichlet problem with freezing of the coefficients. Letting $w \in H W^{1, p}\left(B_{2 R}\right)$ as weak solution of (3.15), we consider

$$
\left\{\begin{array}{l}
\operatorname{div}_{H} a\left(x_{0}, \mathfrak{X} v\right)=0 \quad \text { in } B_{R}, \\
v-w \in H W_{0}^{1, p}\left(B_{R}\right) .
\end{array}\right.
$$

Lemma 3.2. Given weak solution $w \in H W^{1, p}\left(B_{2 R}\right)$ of (3.15), if $v \in H W^{1, p}\left(B_{R}\right)$ is the weak solution of Eq. (3.23), then there exists $c=c(n, p, L)>0$ such that

$$
f_{B_{R}}|\mathfrak{X} v-\mathfrak{X} w|^{p} d x \leq c L^{\prime 2} R^{2 \alpha} f_{B_{R}}(|\mathfrak{X} w|+s)^{p} d x .
$$

Proof. First, note that by testing Eq. (3.23) with $w-v$ and using the ellipticity (2.9b), it is not difficult to show the following inequality,

$$
\int_{B_{R}}|\mathfrak{X} v|^{p} d x \leq c \int_{B_{R}}(|\mathfrak{X} w|+s)^{p} d x
$$

for some $c=c(n, p, L)$; the proof is standard. Also, testing both Eqs. (3.15) and (3.23) with $w-v$, we have that

$$
\int_{B_{2 R}}\langle a(x, \mathfrak{X} w), \mathfrak{X} w-\mathfrak{X} v\rangle d x=0=\int_{B_{R}}\left\langle a\left(x_{0}, \mathfrak{X} v\right), \mathfrak{X} w-\mathfrak{X} v\right\rangle d x
$$


Using the above together with (2.9a) and (1.2), we obtain

$$
\begin{aligned}
& c \int_{B_{R}}\left(|\mathfrak{X} w|^{2}+|\mathfrak{X} v|^{2}+s^{2}\right)^{\frac{p-2}{2}}|\mathfrak{X} w-\mathfrak{X} v|^{2} d x \\
\leq & \int_{B_{R}}\left\langle a\left(x_{0}, \mathfrak{X} w\right)-a\left(x_{0}, \mathfrak{X} v\right), \mathfrak{X} w-\mathfrak{X} v\right\rangle d x \\
= & \int_{B_{R}}\left\langle a\left(x_{0}, \mathfrak{X} w\right)-a(x, \mathfrak{X} w), \mathfrak{X} w-\mathfrak{X} v\right\rangle d x \\
\leq & c L^{\prime} R^{\alpha} \int_{B_{R}}\left(|\mathfrak{X} w|^{2}+|\mathfrak{X} v|^{2}+s^{2}\right)^{\frac{p-2}{2}}|\mathfrak{X} w||\mathfrak{X} w-\mathfrak{X} v| d x .
\end{aligned}
$$

Using Young's inequality on the last integral of the above, it is easy to get

$$
\begin{aligned}
& \int_{B_{R}}\left(|\mathfrak{X} w|^{2}+|\mathfrak{X} v|^{2}+s^{2}\right)^{\frac{p-2}{2}}|\mathfrak{X} w-\mathfrak{X} v|^{2} d x \\
\leq & c\left(L^{\prime} R^{\alpha}\right)^{2} \int_{B_{R}}\left(|\mathfrak{X} w|^{2}+|\mathfrak{X} v|^{2}+s^{2}\right)^{\frac{p}{2}} d x .
\end{aligned}
$$

This, together with (3.25), is enough to prove (3.24).

Combining Lemma 3.1 and Lemma 3.2, we obtain the following comparison estimate of weak solution $u$ of (1.1) and weak solution $v$ of (3.23).

Corollary 3.1. Let $u \in H W^{1, p}(\Omega)$ be a weak solution of $E q$. (1.1) and let $v \in H W^{1, p}\left(B_{R}\right)$ be the weak solution of $E q$. (3.23), where $w \in H W^{1, p}\left(B_{2 R}\right)$ given in the problem (3.23) is the weak solution of Eq. (3.15). Then there exists $c=c(n, p, L)>0$ such that

$$
f_{B_{R}}|\mathfrak{X} v-\mathfrak{X} u| d x \leq c\left(1+\left(L^{\prime} R^{\alpha}\right)^{\frac{2}{p}}\right)\left(\frac{|\mu|\left(B_{2 R}\right)}{R^{Q-1}}\right)^{\frac{1}{p-1}}+c\left(L^{\prime} R^{\alpha}\right)^{\frac{2}{p}} f_{B_{2 R}}(|\mathfrak{X} u|+s) d x .
$$

Proof. First, notice that Hölder's inequality and (3.24) imply

$$
f_{B_{R}}|\mathfrak{X} v-\mathfrak{X} w| d x \leq c\left(L^{\prime} R^{\alpha}\right)^{\frac{2}{p}}\left(f_{B_{R}}(|\mathfrak{X} w|+s)^{p} d x\right)^{\frac{1}{p}} .
$$

Hence, using (3.16) and (3.26), we obtain

$$
\begin{aligned}
f_{B_{R}}|\mathfrak{X} v-\mathfrak{X} u| d x & \leq f_{B_{R}}|\mathfrak{X} w-\mathfrak{X} u| d x+f_{B_{R}}|\mathfrak{X} v-\mathfrak{X} w| d x \\
& \leq c\left(\frac{|\mu|\left(B_{2 R}\right)}{R^{Q-1}}\right)^{\frac{1}{p-1}}+c\left(L^{\prime} R^{\alpha}\right)^{\frac{2}{p}}\left(f_{B_{R}}(|\mathfrak{X} w|+s)^{p} d x\right)^{\frac{1}{p}} .
\end{aligned}
$$


We estimate the last integral using sub-elliptic reverse Hölder's inequality and Gehring's lemma, see [20], to obtain

$$
\begin{aligned}
\left(f_{B_{R}}(|\mathfrak{X} w|+s)^{p} d x\right)^{\frac{1}{p}} & \leq c f_{B_{2 R}}(|\mathfrak{X} w|+s) d x \\
& \leq c f_{B_{2 R}}(|\mathfrak{X} u|+s) d x+c f_{B_{2 R}}|\mathfrak{X} u-\mathfrak{X} w| d x \\
& \leq c f_{B_{2 R}}(|\mathfrak{X} u|+s) d x+c\left(\frac{|\mu|\left(B_{2 R}\right)}{R Q-1}\right)^{\frac{1}{p-1}},
\end{aligned}
$$

where the last inequality follows from (3.16). Now it is easy to see that by combining (3.27) and (3.28), the proof is finished.

\section{Proof of the Theorem 1.1}

We shall prove Theorem 1.1 in this section. As before, here we maintain $u \in H W^{1, p}(\Omega)$ as a weak solution of Eq. (1.1) and fix some arbitrary $x_{0} \in \Omega$ and denote the metric balls $B_{\rho}=B_{\rho}\left(x_{0}\right)$ for every $\rho>0$. The comparison estimates of Section 3 shall lead to the necessary estimates for $u$.

With respect to the given data, let us set

$$
\bar{R}=\bar{R}\left(n, p, L, L^{\prime}, \alpha, \operatorname{dist}\left(x_{0}, \partial \Omega\right)\right)>0,
$$

which shall be chosen as small as required as we proceed, finally the minimum of every reductions of $\bar{R}$, is to be considered. Let

$$
\bar{R} \leq \min \left\{1, \frac{1}{2} \operatorname{dist}\left(x_{0}, \partial \Omega\right), L^{\prime-1 / \alpha}\right\}
$$

to begin with, so that for any $R<\bar{R}$, we have $R, L^{\prime} R^{\alpha}<1$ and $B_{R} \subset \Omega$.

The following lemma is a consequence of the uniform Lipschitz estimate (2.14).

Lemma 4.1. For any $0<\rho \leq R \leq \bar{R} / 2$, we have the estimate

$$
\begin{aligned}
\int_{B_{\rho}}(|\mathfrak{X} u|+s) d x \leq c & \left(\frac{\rho}{R}\right)^{Q} \int_{B_{R}}(|\mathfrak{X} u|+s) d x+c R^{Q}\left(\frac{|\mu|\left(B_{2 R}\right)}{R^{Q-1}}\right)^{\frac{1}{p-1}} \\
& +c\left(L^{\prime} R^{\alpha}\right)^{\frac{2}{p}} \int_{B_{2 R}}(|\mathfrak{X} u|+s) d x .
\end{aligned}
$$

Proof. We denote comparison function $v$ as the weak solution of Eq. (3.23), as before. Then we write

$$
\int_{B_{\rho}}(|\mathfrak{X} u|+s) d x \leq \int_{B_{\rho}}(|\mathfrak{X} v|+s) d x+\int_{B_{\rho}}|\mathfrak{X} u-\mathfrak{X} v| d x .
$$


The first term is estimated from (2.14) as

$$
\begin{aligned}
\int_{B_{\rho}}(|\mathfrak{X} v|+s) d x & \leq c\left(\frac{\rho}{R}\right)^{Q} \int_{B_{R}}(|\mathfrak{X} v|+s) d x \\
& \leq c\left(\frac{\rho}{R}\right)^{Q} \int_{B_{R}}(|\mathfrak{X} u|+s) d x+c\left(\frac{\rho}{R}\right)^{Q} \int_{B_{R}}|\mathfrak{X} v-\mathfrak{X} u| d x .
\end{aligned}
$$

The last terms of (4.3) and (4.4) are estimated by Corollary 3.1 and we end up with (4.2). This concludes the proof.

The following Lemma is similar to a well-known lemma of Campanato [3,10]. The proof follows along the same lines as in [9, Lemma 2.1].

Lemma 4.2. Let $\phi:(0, \infty) \rightarrow[0, \infty)$ be a non-decreasing functions, $A>1$ and $\epsilon \geq 0$ be fixed constants. Let $\psi, \Psi:(0, \infty) \rightarrow[0, \infty)$ be functions such that

$$
\sum_{j=0}^{\infty} \psi\left(t^{j} r\right) \leq \Psi(r)
$$

for any $0<t<t_{0}<1$. Given any $a>0$, suppose that

$$
\phi(\rho) \leq A\left[\left(\frac{\rho}{r}\right)^{a}+\epsilon\right] \phi(r)+r^{a} \psi(r)
$$

holds for any $0<\rho<r \leq R_{0}$, then there exists constants $\epsilon_{0}=\epsilon_{0}(A, a)>0$ and $c=c(A, a)>$ 0 such that if $\epsilon \leq \epsilon_{0}$, then for all $0<\rho<r \leq R_{0}$, we have

$$
\phi(\rho) \leq c\left[\left(\frac{\rho}{r}\right)^{a-\bar{\varepsilon}} \phi(r)+\rho^{a-\bar{\varepsilon}} r^{\bar{\varepsilon}} \Psi(r)\right]
$$

for any $0<\bar{\varepsilon}<a$.

Proof. We fix $0<r \leq R_{0}$. Notice that, for any $0<t<1$, (4.5) implies

$$
\phi(t r) \leq A t^{a}\left(1+\frac{\epsilon}{t^{a}}\right) \phi(r)+r^{a} \psi(r) .
$$

We fix some $t<t_{0}$ and let $\epsilon_{0} \leq t^{a}$, so that for every $\epsilon \leq \epsilon_{0}$, we have

$$
A t^{a}\left(1+\epsilon / t^{a}\right) \leq 2 A t^{a}
$$

Using this on the above, we get

$$
\phi(t r) \leq 2 A t^{a} \phi(r)+r^{a} \psi(r) .
$$


Now, we iterate (4.7) as follows; for any $k \in \mathbb{N}$,

$$
\begin{aligned}
\phi\left(t^{k+1} r\right) & \leq 2 A t^{a} \phi\left(t^{k} r\right)+t^{k a} r^{a} \psi\left(t^{k} r\right) \\
& \leq\left(2 A t^{a}\right)^{2} \phi\left(t^{k-1} r\right)+2 A t^{k a} r^{a} \psi\left(t^{k-1} r\right)+t^{k a} r^{a} \psi\left(t^{k} r\right) \\
& \leq\left(2 A t^{a}\right)^{3} \phi\left(t^{k-2} r\right)+(2 A)^{2} t^{k a} r^{a} \psi\left(t^{k-2} r\right)+2 A t^{k a} r^{a} \psi\left(t^{k-1} r\right)+t^{k a} r^{a} \psi\left(t^{k} r\right) \\
& \leq \cdots \leq\left(2 A t^{a}\right)^{k+1} \phi(r)+t^{k a} r^{a} \sum_{j=0}^{k}(2 A)^{j} \psi\left(t^{k-j} r\right) .
\end{aligned}
$$

Since, $\sum_{j=0}^{\infty} \psi\left(t^{j} r\right) \leq \Psi(r)$ as given, we have

$$
\phi\left(t^{k+1} r\right) \leq\left(2 A t^{a}\right)^{k+1} \phi(r)+\left(2 A t^{a}\right)^{k} r^{a} \Psi(r) .
$$

Now, given any $0<\bar{\varepsilon}<a$, we can choose $t$ small enough such that $t^{\bar{\varepsilon}} \leq \frac{1}{2 A}$ and hence $2 A t^{a} \leq t^{a-\bar{\varepsilon}}$. Then, we have

$$
\phi\left(t^{k+1} r\right) \leq c\left[t^{(k+1)(a-\bar{\varepsilon})} \phi(r)+t^{k(a-\bar{\varepsilon})} r^{a} \Psi(r)\right]
$$

for some $c=c(A, a)>0$. Now, given any $\rho<r$, we can choose $k \in \mathbb{N}$ such that, we have $t^{k+1} r<\rho \leq t^{k} r$. Then, (4.8) implies

$$
\phi(t \rho) \leq \phi\left(t^{k+1} r\right) \leq c\left(\frac{\rho}{r}\right)^{a-\bar{\varepsilon}} \phi(r)+\frac{c}{t^{a-\bar{\varepsilon}}} \rho^{a-\bar{\varepsilon}} r^{\bar{\varepsilon}} \Psi(r),
$$

which, with a rescaling of $\rho$ by constants dependent on $A$, $a$, yields (4.6). This completes the proof.

Using the above Lemma together with Lemma 4.1, we obtain an almost-Lipschitz estimate, as follows.

Proposition 4.1. There exists $c=c(n, p, L)>0$ such that,

$$
\int_{B_{r}}(|\mathfrak{X} u|+s) d x \leq c\left(\frac{r}{R}\right)^{Q-\bar{\varepsilon}}\left[\int_{B_{R}}(|\mathfrak{X} u|+s) d x+R^{Q} \boldsymbol{W}_{\frac{1}{p}, p}^{\mu}\left(x_{0}, R\right)\right]
$$

holds for any $0<\bar{\varepsilon}<Q$ and $0<r \leq R \leq \bar{R}$.

Proof. First, let us fix $0<r \leq \bar{R}$ and denote

$$
\phi(r)=\int_{B_{r}}(|\mathfrak{X} u|+s) d x \quad \text { and } \quad w_{\mu}(r)=\left(\frac{|\mu|\left(B_{r}\right)}{r^{Q-1}}\right)^{\frac{1}{p-1}} .
$$

We recall (4.2) with appropriate scaling, to have

$$
\phi(\rho) \leq c\left(\frac{\rho}{r}\right)^{Q} \phi(r)+c r Q^{Q} w_{\mu}(r)+\left(L^{\prime} r^{\alpha}\right)^{\frac{2}{p}} \phi(r),
$$


for any $\rho \leq r$ and $c=c(n, p, L)>0$. We can apply Lemma 4.2 on the above with $a=Q$ and using approriate reduction

$$
\left(L^{\prime} \bar{R}^{\alpha}\right)^{\frac{2}{p}} \leq \epsilon_{0}(n, p, L) .
$$

Recalling (2.17), notice that $w_{\mu}$ satisfy the summability condition of Lemma 4.2 and we obtain

$$
\phi(r) \leq c\left[\left(\frac{r}{R}\right)^{Q-\bar{\varepsilon}} \phi(R)+r^{Q-\bar{\varepsilon}} R^{\bar{\varepsilon}} \boldsymbol{W}_{\frac{1}{p}, p}^{\mu}\left(x_{0}, R\right)\right]
$$

for every $0<r \leq R \leq \bar{R}$, and hence we have (4.9). This completes the proof.

Now, we use the estimate (3.14) along with the above estimates to prove $C^{1, \gamma}$ regularity of $u$. We continue to assume $\bar{R}$ subject to reductions with dependence of data as in (4.1). First, we have the following lemma.

Lemma 4.3. There exist $\beta=\beta(n, p, L) \in(0,1)$ and $c=c(n, p, L)>0$ such that, for every $0<\varrho<R<\bar{R} / 2$, the following estimate holds:

$$
\begin{aligned}
& f_{B_{Q}}\left|\mathfrak{X} u-(\mathfrak{X} u)_{B_{\varrho}}\right| d x \\
\leq & c\left(\frac{\varrho}{R}\right)^{\beta} f_{B_{R}}(|\mathfrak{X} u|+s) d x+c\left(\frac{R}{\varrho}\right)^{Q}\left[\left(\frac{|\mu|\left(B_{2 R}\right)}{R^{Q-1}}\right)^{\frac{1}{p-1}}+\left(L^{\prime} R^{\alpha}\right)^{\frac{2}{p}} f_{B_{2 R}}(|\mathfrak{X} u|+s) d x\right] .
\end{aligned}
$$

Proof. We define the comparison functions $w$ and $v$ as weak solutions of Eqs. (3.15) and (3.23), as before. Then we have

$$
\begin{aligned}
f_{B_{Q}}\left|\mathfrak{X} u-(\mathfrak{X} u)_{B_{Q}}\right| d x & \leq 2 f_{B_{Q}}\left|\mathfrak{X} u-(\mathfrak{X} v)_{B_{Q}}\right| d x \\
& \leq 2 f_{B_{Q}}\left|\mathfrak{X} v-(\mathfrak{X} v)_{B_{Q}}\right| d x+2 f_{B_{Q}}|\mathfrak{X} u-\mathfrak{X} v| d x .
\end{aligned}
$$

Now, we shall estimate both terms of the right hand side of (4.10) seperately.

Using (3.14), we estimate the first term of (4.10) as

$$
\begin{aligned}
f_{B_{\varrho}}\left|\mathfrak{X} v-(\mathfrak{X} v)_{B_{\varrho}}\right| d x & \leq c\left(\frac{\varrho}{R}\right)^{\beta} f_{B_{R}}(|\mathfrak{X} v|+s) d x \\
& \leq c\left(\frac{\varrho}{R}\right)^{\beta} f_{B_{R}}(|\mathfrak{X} u|+s) d x+c\left(\frac{\varrho}{R}\right)^{\beta} f_{B_{R}}|\mathfrak{X} v-\mathfrak{X} u| d x .
\end{aligned}
$$

The second term of (4.10) is estimated simply as

$$
f_{B_{Q}}|\mathfrak{X} u-\mathfrak{X} v| d x \leq c\left(\frac{R}{\varrho}\right)^{Q} f_{B_{R}}|\mathfrak{X} u-\mathfrak{X} v| d x .
$$

Using the above estimates in (4.10), together with Corollary 3.1 to estimate the integral of $|\mathfrak{X} u-\mathfrak{X} v|$, the proof is finished. 
Now we are ready to prove Theorem 1.1. An extra dependence on $q$ is assumed on $\bar{R}$, where $q>Q$ is as in the statement of Theorem 1.1.

Proof of Theorem 1.1. Let us assume the notation $w_{\mu}$ for the density of the Wolff potential, as used in the previous subsection. From Lemma 4.3, we get

$$
\begin{aligned}
& \int_{B_{Q}}\left|\mathfrak{X} u-(\mathfrak{X} u)_{B_{Q}}\right| d x \\
\leq & c\left(\frac{\varrho}{r}\right)^{Q+\beta} \int_{B_{r}}(|\mathfrak{X} u|+s) d x+c r^{Q} w_{\mu}(r)+c\left(L^{\prime} r^{\alpha}\right)^{\frac{2}{p}} \int_{B_{r}}(|\mathfrak{X} u|+s) d x
\end{aligned}
$$

and from (4.9) of Proposition 4.1, we have,

$$
\int_{B_{r}}(|\mathfrak{X} u|+s) d x \leq c\left(\frac{r}{R}\right)^{Q-\bar{\varepsilon}}\left[\int_{B_{R}}(|\mathfrak{X} u|+s) d x+R^{Q} \boldsymbol{W}_{\frac{1}{p}, p}^{\mu}\left(x_{0}, R\right)\right] .
$$

We use (4.12) on (4.11) to obtain the following estimate,

$$
\begin{gathered}
\int_{B_{\varrho}}\left|\mathfrak{X} u-(\mathfrak{X} u)_{B_{\varrho}}\right| d x \leq c\left(\frac{\varrho^{Q+\beta} R^{\bar{\varepsilon}}}{r^{\beta+\bar{\varepsilon}} R^{Q}}\right)\left[\int_{B_{R}}(|\mathfrak{X} u|+s) d x+R^{Q} \boldsymbol{W}_{\frac{1}{p}, p}^{\mu}\left(x_{0}, R\right)\right] \\
+c r^{Q}\left[w_{\mu}(r)+\left(L^{\prime} r^{\alpha}\right)^{\frac{2}{p}} f_{B_{r}}(|\mathfrak{X} u|+s) d x\right]
\end{gathered}
$$

for every $0<\varrho \leq r \leq R \leq \bar{R}$. Now, given $\mu=f \in L_{\text {loc }}^{q}(\Omega)$ for some $q>Q$, then by Hölder's inequality we have

$$
\frac{|\mu|\left(B_{r}\right)}{r^{Q-1}}=\frac{1}{r^{Q-1}} \int_{B_{r}}|f| d x \leq \frac{\left|B_{r}\right|^{1-1 / q}}{r^{Q-1}}\left(\int_{B_{r}}|f|^{q} d x\right)^{\frac{1}{q}} \leq c r^{1-\frac{Q}{q}}\|f\|_{L^{q} .}
$$

Letting $\delta \leq(1-Q / q) /(p-1)$, the above implies that

$$
w_{\mu}(r) \leq c r^{\delta}\|f\|_{L^{q}}^{1 /(p-1)}
$$

and $0<\delta<1$ since $q>Q$ and $p \geq 2$. The same upper bound is also satisfied by the Wolff potential due to (2.17). Furthermore, we assume $\bar{\varepsilon}<2 \alpha / p$ and $\delta<2 \alpha / p-\bar{\varepsilon}$ so that using (4.9) again for the last term of (4.13) and a further reduction

$$
L^{\prime} \bar{R}^{\alpha-p(\delta+\bar{\varepsilon}) / 2}<1
$$

leads to

$$
\int_{B_{\varrho}}\left|\mathfrak{X} u-(\mathfrak{X} u)_{B_{\varrho}}\right| d x \leq c\left(\frac{\varrho^{Q+\beta}}{r^{\beta+\bar{\varepsilon}}}+r^{Q+\delta}\right)\left[f_{B_{R}}(|\mathfrak{X} u|+s) d x+\|f\|_{L^{q}}^{1 /(p-1)}\right]
$$


for every $0<\varrho \leq r \leq R \leq \bar{R}$. For some $0<\kappa<1$ we rewrite the above with the choice $r=\varrho^{\kappa}$ to have

$$
\begin{aligned}
\int_{B_{\varrho}}\left|\mathfrak{X} u-(\mathfrak{X} u)_{B_{\varrho}}\right| d x & \leq c\left(\varrho^{Q+(1-\kappa) \beta-\kappa \bar{\varepsilon}}+\varrho^{\kappa(Q+\delta)}\right)\left[f_{B_{R}}(|\mathfrak{X} u|+s) d x+\|f\|_{L^{q}}^{1 /(p-1)}\right] \\
& \leq c \varrho^{Q+\gamma}\left[f_{B_{R}}(|\mathfrak{X} u|+s) d x+\|f\|_{L^{q}}^{1 /(p-1)}\right],
\end{aligned}
$$

where the latter inequality follows when $Q+\gamma \leq \min \{Q+(1-\kappa) \beta-\kappa \bar{\varepsilon} \kappa(Q+\delta)\}$; indeed we can make sure that this is true with the choice of $\kappa=\kappa(\gamma)$ such that

$$
\frac{Q+\gamma}{Q+\delta} \leq \kappa \leq \frac{\beta-\gamma}{\beta+\bar{\varepsilon}^{\prime}}
$$

for any $0<\gamma<\beta \delta /(Q+\beta+\delta+\bar{\varepsilon})$. Also, note that if $\gamma, \bar{\varepsilon}$ are small enough, $\kappa=\kappa(\gamma)$ can be chosen close enough to 1 and we can make sure $\varrho^{\kappa} \leq R$, whenever $0<\varrho<R$. Thus, we have obtained

$$
f_{B_{\varrho}}\left|\mathfrak{X} u-(\mathfrak{X} u)_{B_{\varrho}}\right| d x \leq c \varrho^{\gamma}\left[f_{B_{R}}(|\mathfrak{X} u|+s) d x+\|f\|_{L^{q}}^{1 /(p-1)}\right]
$$

for any $0<\varrho<R \leq \bar{R}$ and the proof is completed.

\section{Acknowledgements}

S. Mukherjee was partially supported by "Variationaaliset integraalit geometr" and GHAIA Marie Skłodowska-Curie grant agreement No. 777822 under Horizon 2020. Y. Sire is partially supported by the Simons foundation. This work was initiated at the occasion of a visit of S.M. at Johns Hopkins University, whose hospitality is acknowledged. The authors are thankful to Agnid Banerjee for fruitful discussions and suggestions.

\section{References}

[1] Lucio Boccardo and Thierry Gallouët, Nonlinear elliptic and parabolic equations involving measure data, J. Funct. Anal., 87(1) (1989), 149-169.

[2] A. Bonfiglioli, E. Lanconelli, and F. Uguzzoni, Stratified Lie groups and potential theory for their sub-Laplacians, Springer Monographs in Mathematics, Springer, Berlin, 2007.

[3] Sergio Campanato, Equazioni ellittiche del $\mathrm{II}^{\circ}$ ordine e spazi $\mathcal{L}^{(2, \lambda)}$, Ann. Mat. Pura Appl., 69 (1965), 321-381.

[4] Luca Capogna, Donatella Danielli, and Nicola Garofalo, An embedding theorem and the Harnack inequality for nonlinear subelliptic equations, Commun. Partial Differential Equations, 18(9-10) (1993), 1765-1794.

[5] Luca Capogna, Donatella Danielli, Scott D. Pauls, and Jeremy T. Tyson, An Introduction to the Heisenberg Group and the Sub-Riemannian Isoperimetric Problem, volume 259 of Progress in Mathematics, Birkhäuser Verlag, Basel, 2007. 
[6] E. DiBenedetto, $C^{1+\alpha}$ local regularity of weak solutions of degenerate elliptic equations, Nonlinear Anal., 7(8) (1983), 827-850.

[7] Frank Duzaar and Giuseppe Mingione, Gradient estimates via non-linear potentials, Amer. J. Math., 133(4) (2011), 1093-1149.

[8] G. B. Folland and Elias M. Stein, Hardy Spaces on Homogeneous Groups, volume 28 of Mathematical Notes, Princeton University Press, Princeton, N.J. University of Tokyo Press, Tokyo, 1982.

[9] Mariano Giaquinta, Multiple Integrals in the Calculus of Variations and Nonlinear Elliptic Systems, volume 105 of Annals of Mathematics Studies, Princeton University Press, Princeton, NJ, 1983.

[10] Mariano Giaquinta and Enrico Giusti, Sharp estimates for the derivatives of local minima of variational integrals, Boll. Un. Mat. Ital. A, 3(2) (1984), 239-248.

[11] Enrico Giusti, Direct Methods in the Calculus of Variations, World Scientific Publishing Co., Inc., River Edge, NJ, 2003.

[12] David Jerison, The Poincaré inequality for vector fields satisfying Hörmander's condition, Duke Math. J., 53(2) (1986), 503-523.

[13] Tero Kilpeläinen and Jan Malý, The Wiener test and potential estimates for quasilinear elliptic equations, Acta Math., 172(1) (1994), 137-161.

[14] David Kinderlehrer and Guido Stampacchia, An Introduction to Variational Inequalities and Their Applications, volume 31 of Classics in Applied Mathematics, Society for Industrial and Applied Mathematics (SIAM), Philadelphia, PA, 2000. Reprint of the 1980 original.

[15] John L. Lewis, Regularity of the derivatives of solutions to certain degenerate elliptic equations, Indiana Univ. Math. J., 32(6) (1983), 849-858.

[16] Gary M. Lieberman, The natural generalization of the natural conditions of Ladyzhenskaya and Ural'tseva for elliptic equations, Commun. Partial Differential Equations, 16(2-3) (1991), 311-361.

[17] S. Mukherjee and X. Zhong, $C^{1, \alpha}$-Regularity for variational problems in the Heisenberg group, Anal. PDE., 14(2) (2021), pp. 567-594.

[18] Shirsho Mukherjee, On local Lipschitz regularity of Quasilinear Elliptic equations in the Heisenberg Group, Nonlinear Anal., 212 (2021), 112453.

[19] Peter Tolksdorf, Regularity for a more general class of quasilinear elliptic equations, J. Differential Equations, 51(1) (1984), 126-150.

[20] Anna Zatorska-Goldstein, Very weak solutions of nonlinear subelliptic equations, Ann. Acad. Sci. Fenn. Math., 30(2) (2005), 407-436.

[21] Xiao Zhong, Regularity for variational problems in the Heisenberg Group, https: //arxiv . org/abs/1711.03284, 2017. 